\title{
3D morphology of the PN IRAS 18333-2357
}

\section{Muthumariappan}

Indian Institute of Astrophysics, Bangalore 560 034, India. email: muthu@iiap.res.in

\begin{abstract}
We analysed the Spitzer $8 \mu \mathrm{m}$ and WISE $22 \mu \mathrm{m}$ images of the PN IRAS 18333-2357 using a 3D RT code. We describe its morphology with a H-poor disk and a spherical shell around it.
\end{abstract}

Keywords. (ISM:) planetary nebulae: individual (IRAS 18333-2357)

\section{Introduction}

The Halo PN IRAS 18333-2357 is a strong IR source located in the globular cluster M22. It is important in understanding the evolution of low mass stars, stellar merger at low metallicity and the dust grains ejected from metal-poor systems. The space velocity of M22 is $158 \mathrm{~km} \mathrm{~s}^{-1}$ and the ram pressure causes a asymmetry in the PN morphology. The nebular spectrum has forbidden lines but no $\mathrm{H}$ and He lines, showing It's H-poor nature with a lack of photo-ionization and presence of dust heating (Borkowski \& Harrington(1991)). The nebula displays carbon rich dust chemistry (Muthumariappan et al. $(2013))$.

\section{Radiative Transfer Model and Results}

We used archival UV (FUSE), optical (Palomar), near-IR (2MASS and DENIS, mid- and far-IR (WISE, IRAS and Akari) photometric and Spitzer-IRS spectroscopic observations to construct its SED from far-UV to the far-IR. Spitzer $8 \mu \mathrm{m}$ and WISE $22 \mu \mathrm{m}$ images are used to trace the emission from PAH and very small grains (VSG, $\leqslant$ $200 \AA$ ) and continuum from cool dust respectively. The SED and the thermal images were modelled using a 3D Monte-Carlo RT Code Hochunk-3D to derive the nebular morphological parameters. The shell has a $1 / \mathrm{r}^{n}$ density function and an equatorial torus is (at $40^{\circ}$ inclination) at the center. Thermally fluctuating grains are included in the radiative transfer. The best-fit model SED is presented in Fig. 1, and the images are shown in Fig 2.

Excess emission seen in the $3-12 \mu \mathrm{m}$ region of SED is due to $\mathrm{PAH} / \mathrm{VSG}$ which arise strongly from the disk. Disk's sky- projected sizes overlap with the optical nebula showing their co-existence, supporting nubular heating by photo-elecric emission from PAH/VSG. The central star temperature is $50000 \mathrm{~K}$ and it's luminosity is $2640 \mathrm{~L}_{\odot}$. Nebular gas to dust mass ratio is seven. The morphology of IRAS 18333-2357, it's dust mass and it's gas to dust mass ratio (Table 1) are similar to the H-poor inner ejecta of PN A30. This similarity suggests that they possibly share similar origin: PN formed from born-again event. WISE $22 \mu \mathrm{m}$ image shows a faint, half moon shaped, fragmented outer envelope which is nearly perpendicular to the direction of the cluster's motion. This could be due to the PN's interaction with the Halo gas. This faint envelope could be the ejection before the Late thermal pulse and it likely to have untraced H-rich gas. 
Table 1. Dust shell parameters

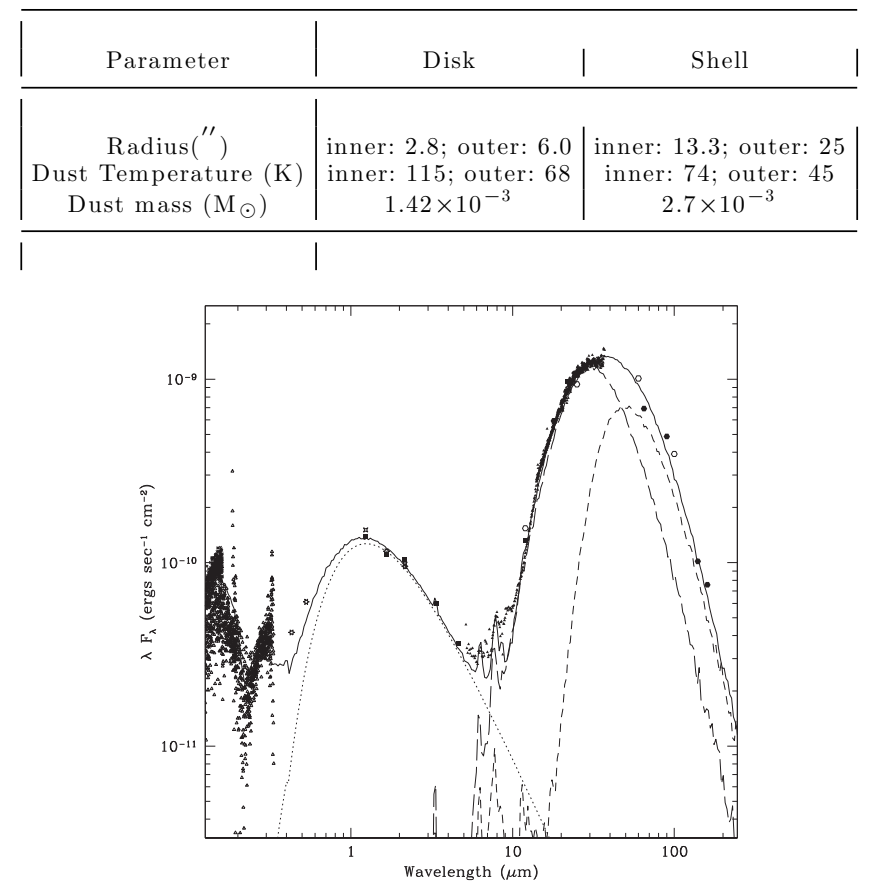

Figure 1. SED fit (solid line) to the observation. Flux from the disk (long-dashed line), from the shell (short-dashed line) and field star (dotted line) are also shown. Observations: HST FOS spectrum (diaginal cross), IUE spectrum (open triangles), DENIS (filled stars), WISE (filled squares), Spitzer IRS (filled triangles), IRAS (open hexagons) and Akari (filled hexagon).
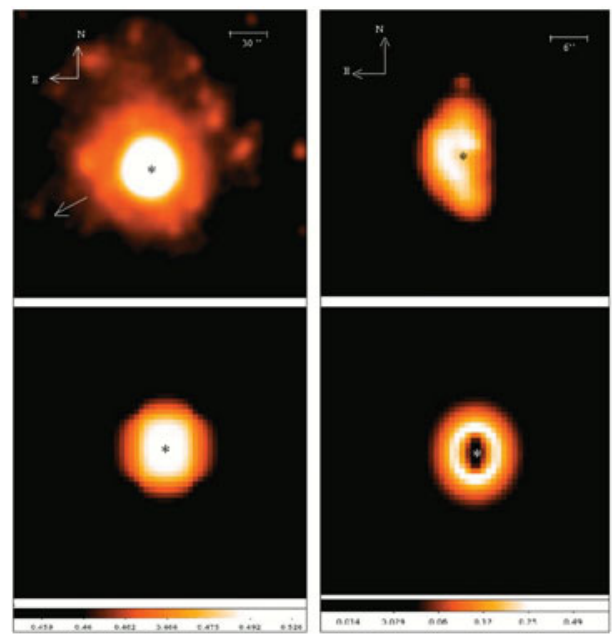

Figure 2. Top Panels: WISE $22 \mu \mathrm{m}$ (left) and Spitzer IRAC $8 \mu \mathrm{m}$ (right) images. Bottom Panels: model images at $22-$ and $8 \mu \mathrm{m}$. The PN motion in the sky is shown (top left picture).

\section{References}

Borkowski K. J. \& Harrington J. P. 1991, ApJ, 379, 168

Muthumariappan C., Parthasarathy, M., \& Ita Y. 2013, MNRAS, 435, 60 